Andreev, Oleg; Kolesnikov, Yurii; Thess, André:

Visualization of the Ludford column

URN: $\quad$ urn:nbn:de:gbv:ilm1-2015210138

Published OpenAccess: January 2015

Original published in:

Journal of fluid mechanics. - Cambridge [u.a.] : Cambridge Univ. Press (ISSN 1469-7645). - 721 (2013), S. 438-453.

DOI: $\quad 10.1017 / \mathrm{jfm} .2013 .76$

URL: $\quad$ http://dx.doi.org/10.1017/jfm.2013.76

[Visited: 2014-08-27]

„Im Rahmen der hochschulweiten Open-Access-Strategie für die Zweitveröffentlichung identifiziert durch die Universitätsbibliothek IImenau."

"Within the academic Open Access Strategy identified for deposition by IImenau University Library."

„Dieser Beitrag ist mit Zustimmung des Rechteinhabers aufgrund einer (DFG-geförderten) Allianz- bzw. Nationallizenz frei zugänglich."

"This publication is with permission of the rights owner freely accessible due to an Alliance licence and a national licence (funded by the DFG, German Research Foundation) respectively."

\section{DFG}

Nationallizenzen 


\title{
Visualization of the Ludford column
}

\author{
Oleg Andreev $\ddagger$ Yurii Kolesnikov and André Thess $\dagger$ \\ Institute of Thermodynamics and Fluid Mechanics, Ilmenau University of Technology, PO Box 100565, \\ 98684 Ilmenau, Germany
}

(Received 24 February 2012; revised 15 January 2013; accepted 1 February 2013; first published online 13 March 2013)

When a liquid metal flows around a truncated cylinder in the presence of a magnetic field which is parallel to the axis of the cylinder, a stagnant region develops above the cylinder. We call this region a Ludford column. The Ludford column represents the magnetohydrodynamics (MHD) analogue of the well-known Taylor columns in rotating flows. Whereas Taylor columns can be easily visualized using dye, the visualization of Ludford columns has remained elusive up to now because liquid metals are opaque. We demonstrate that this fundamental limitation of experimental MHD can be overcome by using a superconducting $5 \mathrm{~T}$ magnet. This facility permits us to perform MHD experiments in which the opaque liquid metals are replaced with a transparent electrolyte while maintaining the key MHD effects. We report results of a series of flow experiments in which an aqueous solution of sulphuric acid flows around a bar with square cross-section (which for simplicity shall be referred to as a cylinder). We vary the Reynolds number in the range $5<R e<100$ and the Hartmann number in the range $0<H a<14$. The experimental procedure involves flow visualizations using tracer particles as well as velocity measurements using particle image velocimetry (PIV). Our experiments provide direct access to the Ludford column for the first time and reveal the spatial structure of this basic feature of MHD flows.

Key words: high-Hartmann-number flows, MHD and electrohydrodynamics, MHD turbulence

\section{Introduction}

Flows around obstacles are among the most common problems encountered in the fluid mechanics literature, and cylindrical obstacles have received particularly broad attention. The reason is that this relatively simple geometry encompasses many of the important physical effects likely to play a role in flow around more complicated obstacles. This implies that a profound understanding of the flow past a cylinder will facilitate the analysis of a wide variety of applied problems ranging from aerodynamics to pollutant dispersion around buildings and flows in turbines.

When the fluid is electrically conducting such as for instance a liquid metal in metallurgical applications, additional effects appear (Moreau 1990; Davidson 1999; Müller \& Bühler 2010). In particular, the presence of a magnetic field tends to

$†$ Email address for correspondence: thess@tu-ilmenau.de

$\ddagger$ Present address: Institute of Fluid Dynamics, Helmholtz-Zentrum Dresden-Rossendorf, Bautzner Landstraße 400, 01328 Dresden, Germany. 
homogenize the flow along the direction of the magnetic field lines. This leads to strong changes in the flow structures which are qualitatively similar to the formation of the well-known Taylor columns above a truncated obstacle in rotating flows. The goal of the present work is to visualize and investigate the magnetohydrodynamic analogue of Taylor columns (Greenspan 1969) which we shall refer to as Ludford columns.

If the magnetic field in an electrically conducting fluid has the same direction as the axis of a truncated cylinder and the electromagnetic interaction parameter (to be defined below) is sufficiently large, a stagnant region is formed above the cylinder. This stagnant region was first identified by Ludford (1961) who studied magnetohydrodynamic (MHD) flows around obstacles. The theory was further developed by Hunt \& Leibovich (1967) and Hunt \& Ludford (1968). According to their predictions MHD boundary layers are formed along the magnetic field, originating from sharp bends in ducts as well as at points where the duct geometry changes. Since these layers are often referred to as Ludford layers, we shall term the stagnant region above a truncated cylinder a Ludford column, which, as mentioned above, can be regarded as the MHD-counterpart of Taylor columns in rotating systems (Greenspan 1969).

The understanding of Ludford columns, which is the central focus of the present work, is not only interesting in its own right. This configuration is also a generic one as MHD flows around obstacles also occur in a wide variety of applications. For instance, the space vehicle re-entry problem features the flow of conducting plasma around an obstacle. Takizawa et al. (2006) have shown that it could be influenced by a strong magnetic field in order to reduce heat transfer. The cooling blanket of the fusion reactor ITER which is currently under construction in Cadarache (France), features complex flows of liquid metal in magnetic fields of the order of $10 \mathrm{~T}$ in which the occurrence of obstacles and stagnant effects cannot be avoided (Kolesnikov \& Tsinober 1972; Moreau 1990; Kolesnikov \& Andreev 1997). The motivation of the present investigation also comes from the necessity to use MHD effects for control in electromagnetic processing of materials (Moreau 1990; Davidson 1999) as well as MHD separation of particles and liquid metal transport in metallurgy (Davidson 1999).

For MHD processes with liquid metals the value of the Hartmann number, which characterizes the ratio between electromagnetic and viscous forces, is often high. This implies that the electromagnetic forces prevail over the viscous forces, and that such MHD flows can be split into a quasi-inviscid core flow and relatively thin boundary layers or free shear layers of two types. The core occupies almost the whole volume of the flow. In this region there is a balance between a driving force (pressure or externally applied electrical current) and the Lorentz force. In the boundary layer regions, the viscous forces are balanced by the Lorentz forces. The boundary layer adjacent to the wall perpendicular to the magnetic field is called the Hartmann layer and is characterized by an exponential decay of the velocity towards the wall and by thickness of order $\delta_{H} \sim O\left(\mathrm{Ha}^{-1}\right)$ where the Hartmann number $\mathrm{Ha}$ will be defined below (Shercliff 1975). The boundary layer adjacent to the wall parallel to the magnetic field is termed the Shercliff layer. Its thickness scales as $\delta_{S} \sim O\left(H a^{-1 / 2}\right)$ (Shercliff 1953, 1962, 1975).

Theoretically the effect of a magnetic field on the evolution of vortices has been investigated by Sommeria \& Moreau (1982), Davidson (1997) and Pothérat, Sommeria \& Moreau (2000) who showed that the velocity components of three-dimensional perturbations are suppressed by Joule dissipation, except in the Hartmann layer where the turbulent fluctuations are weakly damped due to the sharp velocity gradients. The vortices parallel to the magnetic field have a tendency to stretch along the field 
lines. The dissipation of mechanical energy in this case is of viscous nature and is concentrated in the Hartmann boundary layers at the walls perpendicular to the field. The electrical current penetrates into this layer from the core flow in order to maintain the electromagnetic force and balance the viscosity. That is a very general mechanism of exchange of momentum between the core flow and the boundary layers. The magnetic field mainly stabilizes the vortices parallel to the field with minor dissipation compared to the vortices oriented perpendicular to the field. In particular, the orientation of the magnetic field with respect to the axis of a cylindrical obstacle significantly affects the configuration of the vortical structures in the vicinity of and behind the obstacle.

Whereas the case when the magnetic field is oriented along the streamwise direction has received significant attention (Lahjomri, Capéran \& Alémany 1993; Mutschke et al. 1997), the literature on flows where the magnetic field is aligned with the cylinder axis is scarce. Experimentally such flows around a cylinder were investigated by Branover et al. (1969), Kolesnikov \& Tsinober (1972), Josserand, Marty \& Alémany (1993), Andreev \& Kolesnikov (1997) and Frank, Barleon \& Müller (2001). The particular motivation of the present study comes from the observation by Kolesnikov \& Andreev (2000) of a two-step mechanism for the onset of instability past a cylinder in experiments with liquid metal permeated by a strong axial magnetic field. If the length scale $\delta_{S} \sim O\left(\mathrm{Ha}^{-1 / 2}\right)$ of the Shercliff boundary layer becomes smaller than the diameter of the cylinder, the instability occurs first in this layer which contains the cylinder itself and the stable attached vortices. As a result, this flow configuration generates small-scale perturbations upon the onset of instability. This effect defines the first value of the critical Reynolds number. The breakdown of the large-scale attached vortices commensurable with the diameter of the cylinder occurs later with the growth of the inlet velocity and defines the second critical Reynolds number.

In the present work we attempt to visualize this effect. Dousset \& Pothérat $(2008,2012)$ presented a comprehensive review of numerical simulations of MHD flow around a cylinder (in their terminology referred to as a 'truncated cylinder').

Our paper is organized as follows. In $\S 2$ we describe the design of the experimental device and the methods of optical measurements and visualization. In $\S 3$ the results are described: $\S 3.1$ contains the results of the flow visualization, which we performed for the creeping regime at Reynolds number equal to 5. Here the Ludford column is clearly seen above the end face of the moving cylinder in the transparent electrolyte under the imposed axial magnetic field. Vortical structures visualized for a large Reynolds number in the vertical mid-plane parallel to the magnetic field are discussed in §3.2. For the same regimes, the structure distributions in a plane perpendicular to the magnetic field are shown in $\S 3.3$. In $\S 4$ we summarize our conclusions and discuss some future questions that would be useful to investigate.

\section{Experimental method}

\subsection{Test section}

The visualization experiments are performed in an open vessel filled with an aqueous solution of sulphuric acid $\left(\mathrm{H}_{2} \mathrm{SO}_{4}\right)$. At room temperature this transparent electrolyte has a relatively high electrical conductivity compared with other waterbased electrolytes. The physical properties of this solution, taken from Zaytsev \& Aseyev (1992) for a mass concentration of $37 \%$ and at a temperature of $20{ }^{\circ} \mathrm{C}$, are as follows: density $\rho=1222 \mathrm{~kg} \mathrm{~m}^{-3}$, kinematic viscosity $\nu=2.04 \times 10^{-6} \mathrm{~m}^{2} \mathrm{~s}^{-1}$ and electrical conductivity $\sigma=87 \Omega^{-1} \mathrm{~m}^{-1}$. 
(a)

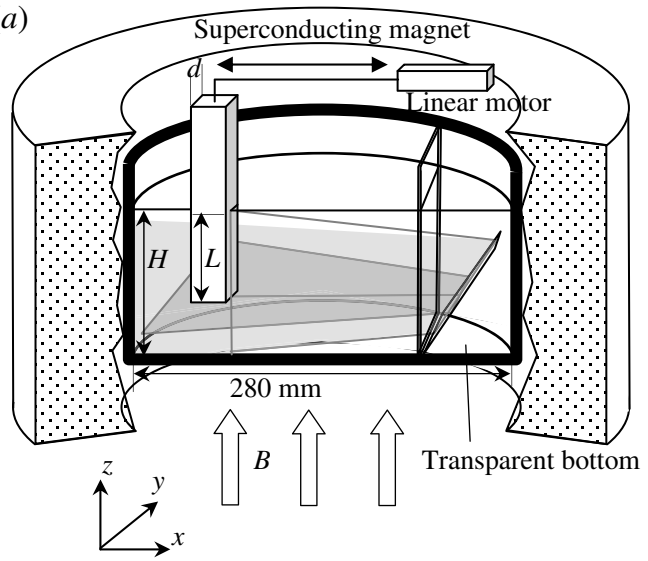

(b)

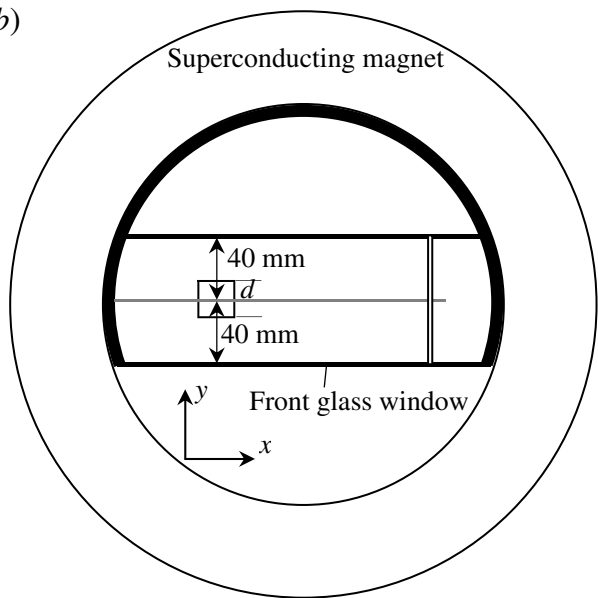

FIGURE 1. Sketch of the experimental set-up: $(a)$ side view, $(b)$ top view. The linear motor moves the cylinder whose lower part is immersed in electrolyte with a wetted length $L=67 \mathrm{~mm}$. The depth of the electrolyte is $H=90 \mathrm{~mm}$, the lower face of the cylinder is $23 \mathrm{~mm}$ above the bottom.

Figure 1 shows a schematic of the experimental set-up. A special vessel with a diameter of $280 \mathrm{~mm}$ and a height of $150 \mathrm{~mm}$ is installed inside the 30 -cm-diameter warm bore of a superconducting magnet. The magnet develops a DC magnetic field whose induction is varied stepwise in the range $B_{0}=0,2,3,4,5 \mathrm{~T}$. The front wall and the bottom wall of the vessel are made of glass and allow access for visualization and optical flow measurement. In the experiments we use a non-conducting bar with square cross-section made of Plexiglas and having a polished surface. For simplicity we shall use the term 'cylinder' instead of 'bar with square cross-section'. This obstacle has the advantage that it does not perturb the light sheet in contrast to a cylinder acting as a lens. The cylinder is moved in the quiescent electrolyte, filled to a level of $H=90 \mathrm{~mm}$, by a linear motor. We use a single light source to illuminate regions both upstream and downstream of the cylinder. The cylinder has a width of $d=15 \mathrm{~mm}$, a total length of $150 \mathrm{~mm}$, and is submerged in the electrolyte to a length of $L=67 \mathrm{~mm}$. The quantity $d$ is used as the length scale for the computation of the non-dimensional parameters. The cylinder is situated at a distance of $40 \mathrm{~mm}$ from the sidewalls. This corresponds to a rather large value of the blockage ratio of $37.5 \%$. This is due to the limited space inside the magnet bore as shown in figure $1(b)$. In the experimental runs the cylinder travels a distance of $240 \mathrm{~mm}$. The Cartesian coordinate system with the origin placed on the top of the cylinder in its centre is defined in figure 2 . The direction of the $x$-coordinate is opposite to the motion of the cylinder and corresponds to the direction of the incoming flow in the coordinate system associated with the cylinder. The experiments are conducted for cylinder velocities in the range $0.7 \leqslant U_{0} \leqslant 13.6 \mathrm{~mm} \mathrm{~s}^{-1}$, corresponding to Reynolds numbers $R e=U_{0} d / v$ in the range $5 \leqslant R e \leqslant 100$. The Hartmann number $H a=B_{0} d(\sigma / v \rho)^{1 / 2}$ which represents the square root of the ratio between the electromagnetic and viscous forces attains a maximum value of $H a=14$ in a magnetic field of $5 \mathrm{~T}$.

\subsection{Visualization and particle image velocimetry (PIV) measurements}

Since commercially available PIV equipment is not designed for operation in the vicinity of a strong magnetic field, a low-cost custom-made optical system was used 
(a)

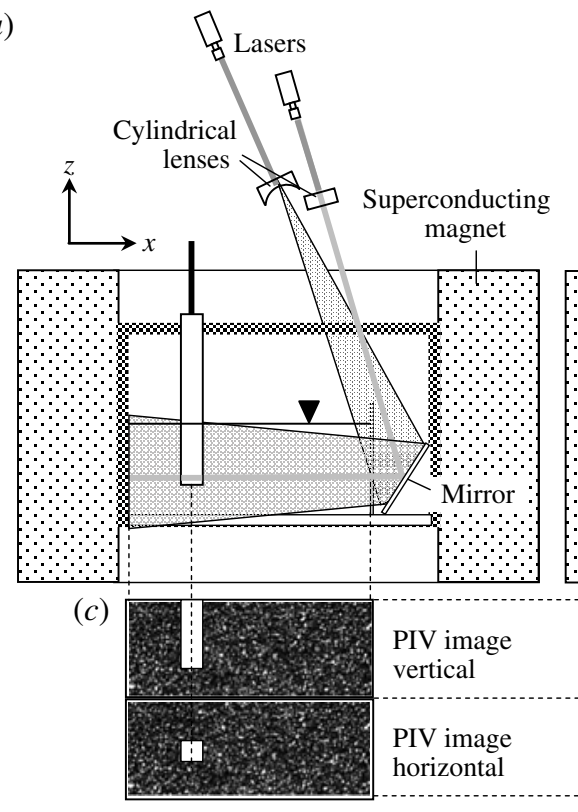

(b)

$\square$ Lasers 


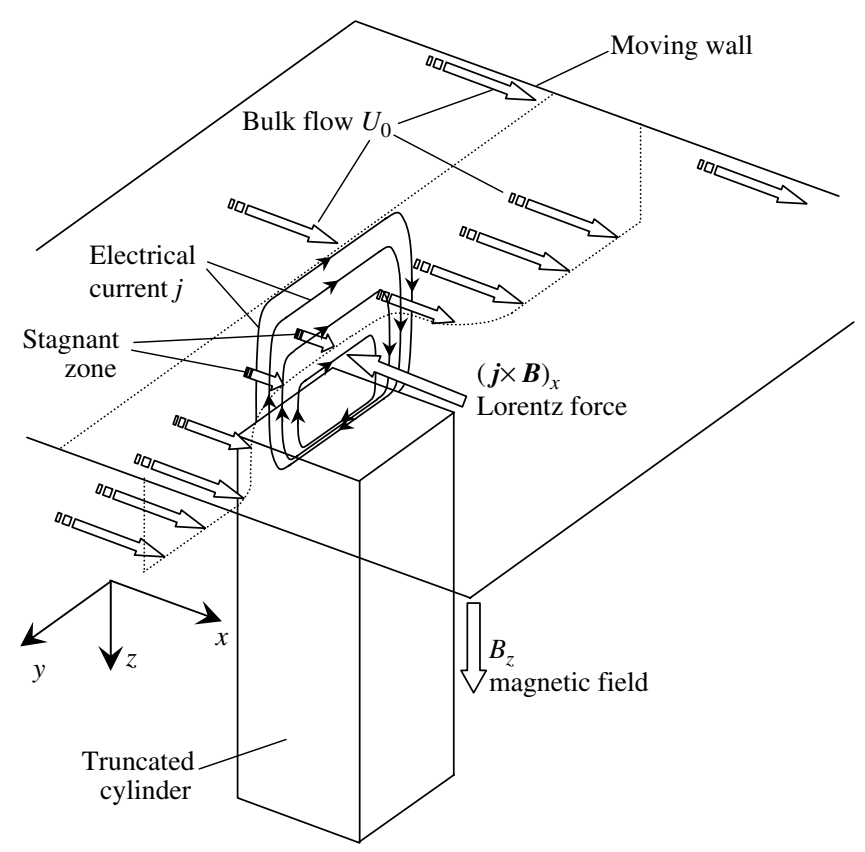

FIGURE 3. Schematic illustration of the formation of the Ludford column as seen from a coordinate system attached to the moving cylinder and turned upside down. In this coordinate system the cylinder is at rest and the bottom of the vessel is represented by a moving plate above the top surface of the cylinder.

To run the program, one needs the MATLAB Image Processing and DACE (Design and Analysis of Computer Experiments) Toolboxes. The latter is available from http:// www.imm.dtu.dk/hbn/dace/.

\section{Results}

\subsection{Flow structures for Re $<20$ and the Ludford column}

The formation mechanism of the Ludford column extending along the magnetic field from the end of the cylinder to the wall is schematically shown in figure 3. For illustration purposes we discuss the results as if the cylinder were at rest and the wall moving with constant velocity. In the presence of the magnetic field the difference in velocities between the fluid adjacent to the surface of the cylinder end and the layer near the wall induces eddy currents as indicated in figure 3. For comparison, for homogeneous motion of the fluid the eddy currents do not appear if the cylinder is absent and there is only the generation of the potential difference in the fluid between sidewalls. The currents parallel to the magnetic field pass through vertical shear layers of thickness $d \mathrm{Ha}^{-1 / 2}$, whereas the currents near the cylinder end flow through the Hartman shear layer of thickness $d \mathrm{Ha}^{-1}$. The interaction of the currents and the magnetic field generates a breaking Lorentz force $f_{x}=(\boldsymbol{j} \times \boldsymbol{B})_{x}$ in the layers above the end of cylinder. In response to this, a stagnant region is produced in the fluid region bounded on the one hand by two vertical shear layers, and on the other hand by the end of the cylinder and the non-conducting wall. The diffusion of momentum from the cylinder along the magnetic field direction becomes more pronounced as the magnetic field grows. Such an effect is most conspicuous at electromagnetic 


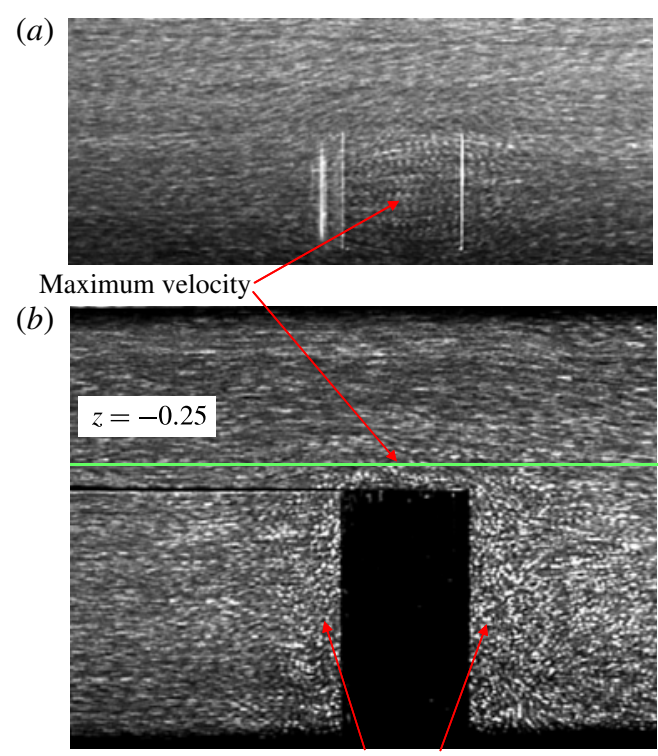

Stagnant zones
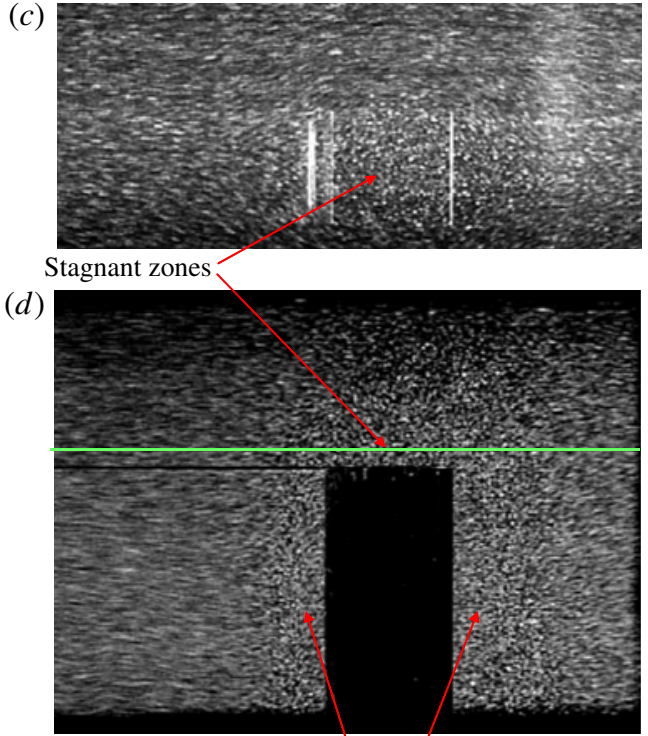

Stagnant zones

FIgURE 4. (Colour online) Visualization of the Ludford column in the two planes $z=-0.25$ and $y=0$ for Reynolds number $R e=5$ with and without magnetic field: $(a, b) H a=0,(c, d)$ $H a=14(N=39.2)$. (Horizontal black line ahead of the cylinder is an optical effect from the end face of the cylinder.)

interaction parameters $N=H a^{2} / R e>1$, which represents the ratio of Lorentz force to inertial force.

We start our discussion of the experimental results with the case of slow motion, when the cylinder does not generate vortex structures. With our electrolyte and magnet our experiments are limited to a maximum value of the Hartmann number of $\mathrm{Ha}=14$. However, we can realize Reynolds numbers in the range of $5<R e<20$ and obtain comparatively high values of the electromagnetic interaction parameters in the range of $9.8<N<39.2$.

Figure 4 demonstrates the results at $\mathrm{Re}=5$ without a magnetic field $(\mathrm{Ha}=0)$ and in the presence of the magnetic field at $H a=14$, i.e. at $N=39.2$ when the stagnant region is pronounced. The flow without a magnetic field shown in figure $4(a, b)$ completely corresponds to the flow around a truncated cylinder in ordinary hydrodynamics. In figure $4(a)$ one can see a weak influence of the cylinder end on the flow at a distance of $z=-0.25$. We should note that for $N<1$ the flow around a cylinder placed in homogeneous flow is also three-dimensional near the cylinder. Figure $4(d)$ demonstrates that in the presence of a high magnetic field ( $B=5 \mathrm{~T}$ and $N=39.2$ ) the flow evolves in such a way as to produce a Ludford column as predicted in earlier work (Ludford 1961; Hunt \& Ludford 1968). Stagnant fluid is clearly observed in figure $4(c)$ inside the projection of the cylinder crosssection on the plane $z=-0.25$. In all images the short white streaks or points visualize the stagnant region. It should be noted that in a strong homogeneous magnetic field $(N>1)$ the flow of conducting fluid has the tendency to be twodimensional in the plane perpendicular to the field. As a consequence, the Lorentz force induced opposite to the flow creates the stagnant region above the cylinder. In 
(a)

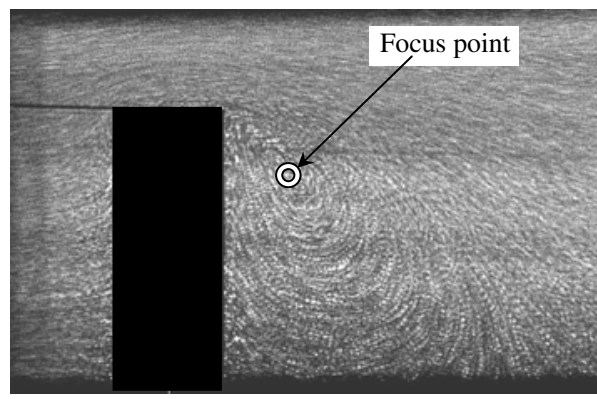

(b)

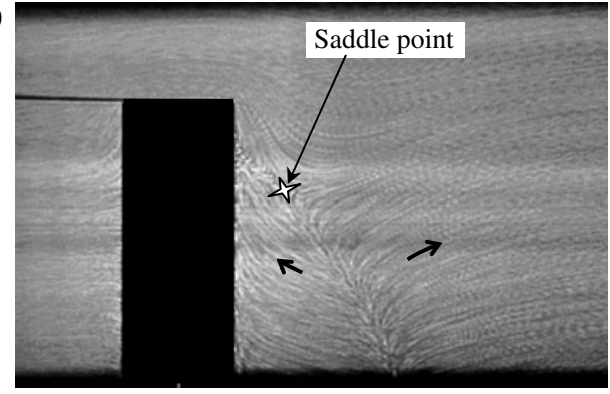

FIGURE 5. Flow structures in the vicinity of the cylinder in the vertical mid-plane at $R e=50$ : (a) $H a=0$ and (b) $H a=14(N=3.92)$. The symbols $\odot$ and $\&$ mark the focus and saddle point respectively. The horizontal black line ahead of the cylinder is a shadow from the top face of the cylinder.

the case when the coordinate system is attached to the moving cylinder this region moves together with the cylinder whereas the surrounding fluid remains at rest.

\subsection{Flow structures for $10 \leqslant R e \leqslant 100$ in the vertical mid-plane parallel to the magnetic field}

The difference in the flow structures past the truncated cylinder at $R e=50$ with and without a magnetic field is shown in figure 5. Without the magnetic field the so-called 'head' vortex (Hunt et al. 1978; Dousset \& Pothérat 2010) is developed and completely occupies the visible region behind the cylinder as one can see in figure 5(a). It should be mentioned that the horseshoe vortex and the base vortices are not observed in our experiments in the absence of a wall boundary layer whose instability could feed these vortices. The head vortex is visualized by a circular system of streaks in the considered plane, i.e. the vortex axis is perpendicular to the magnetic field. The central point is classified as a focus point. If a magnetic field is applied (see figure $5 b$ ), the vortex is broken up by a slowing of the flow above the end of the cylinder (because of $N>1$ ) and, perhaps, partly through the Joule dissipation due to the inner electric currents induced by the perpendicular orientation of the vortex to the magnetic field. Moreover, this value of the Reynolds number is high enough for the generation of attached vortices past the cylinder, which are clearly visible in figure 9 for $R e=50$. The region above the cylinder is occupied by slowly moving fluid (in the limit $N \rightarrow \infty$ the fluid is at rest) and there exists a certain angle between the axes of the vortices and the magnetic field direction under which the Joule dissipation is sufficiently small (in the ideal case of $\operatorname{Re} \neq 0, N \rightarrow \infty$ the dissipation is zero and the flow around a cylinder becomes two-dimensional in the plane perpendicular to the field). In our case of $\mathrm{Ha}=14$ and $\mathrm{Re}=50$ the flow becomes quasi-two-dimensional. In our visualization of streamlines in figure $5(b)$ we have denoted the flow directions by two arrows. The right arrow indicates the outer flow past the ends of the vortices (see also figure 8 for $R e=50$ and figure $9 e$ ). One can see that the attached vortices are deformed in the vertical direction and they possess a non-zero vertical velocity component. In this regime the structure of the flow above the end of the cylinder is evident. The point where the attached vortices contact the outer flow can be identified as a saddle point.

Figure 6 demonstrates the behaviour of the flow for increasing values of the interaction parameter in the range of $0.63 \leqslant N \leqslant 4.18$. For small $N$ and for $\operatorname{Re}=50$ one can see in figure 6(a) that a head vortex is generated by the fluid above the 
(a)

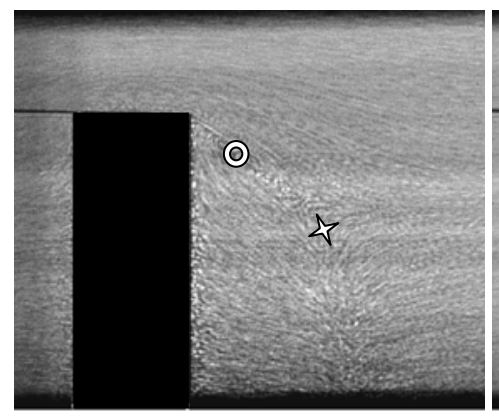

(b)

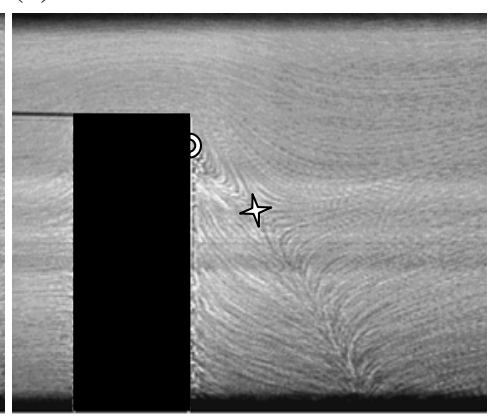

(c)

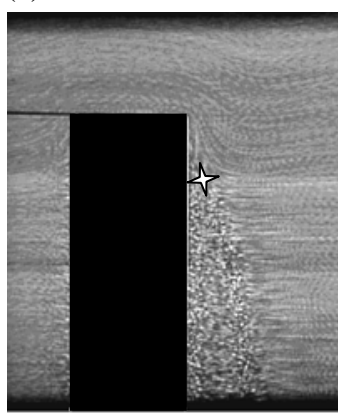

FIgURE 6. Flow in the vicinity of the cylinder and the positions of the saddle $\downarrow$ and focus () points: (a) $R e=50, H a=5.6$ and $N=0.63$; (b) $R e=50, H a=11.2(N=2.51)$; (c) $R e=30, H a=11.2(N=4.18)$. The horizontal black line ahead of the cylinder is a shadow from the top face of the cylinder.

cylinder and that two slightly pronounced attached vortices behind the cylinder emerge with a tendency to be parallel to the magnetic field. The growth of $N$ at constant $R e$ leads to a slowing of the fluid above the cylinder and hence to a decrease of head vortex generation (figure $6 b$ ). In addition, the vortex is suppressed due to the Joule dissipation as already mentioned above. Due to these two causes of a weakening of the head vortex, the attached vortices build up and fill the space behind the cylinder because they possess minimum Joule dissipation (Kolesnikov \& Tsinober 1972; Andreev \& Kolesnikov 1997) when their axes are parallel to the magnetic field. In an early experimental study of the effect of a transverse magnetic field on perturbations in the wake behind a cylinder in a $20 \% \mathrm{KCl}$ solution electrolyte (Branover et al. 1969) it was established that application of the field reduces the intensity of the vortices perpendicular to the field and simultaneously reorients the vortices in the wake so that their axes become parallel to the field. This transformation of motion was observed even at $N=0.26$. When the Hartmann number is kept constant at a value of $H a=11.2$ and the Reynolds number is decreased to 30 so that the electromagnetic interaction parameter exceeds $N=4.18$, the head vortex is no longer observed and the area of attached vortices becomes narrower behind the cylinder, as can be seen in figure 6(c).

In order to classify the flow patterns we present in figure 7 the results of detailed measurements of the dependence of the positions of saddle points behind the cylinder on the values of the Reynolds and Hartmann numbers. To generate the lines, the positions of saddle points were evaluated by averaging over several images using an exposure time of $5 \mathrm{~s}$. The positions were recorded for Reynolds numbers in the ranges $10 \leqslant R e \leqslant 50$ for $H a=0 ; 15 \leqslant R e \leqslant 65$ for $H a=5.6,15 \leqslant R e \leqslant 85$ for $H a=8.4$, $15 \leqslant R e \leqslant 90$ for $H a=11.2$ and $15 \leqslant R e \leqslant 90$ for $H a=14$. The experimental data thus provide the lowest position of the saddle point $S_{C}$ at the highest value of $R e$ and the upper position of the saddle point at the lowest value of $R e$ for all Hartmann numbers presented in this figure. Hence the lower points correspond to small interaction parameter whereas the upper points correspond to large interaction parameters in the range $0.48 \leqslant N \leqslant 13.09$. In the three sketches we also schematically indicate the positions of the focus points for the respective flow regimes. One can see from the figure that the transition regions for all regimes, where the positions 


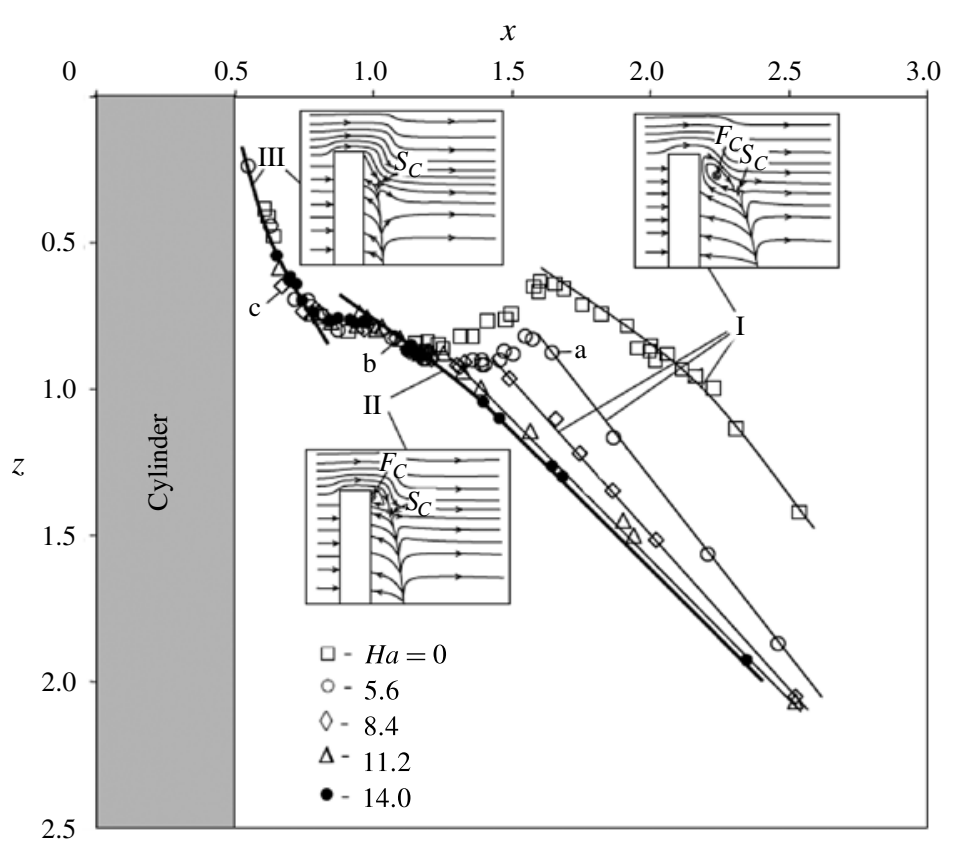

FIGURE 7. Positions of the saddle points $S_{C}$ plotted for different Reynolds numbers in the range $10 \leqslant R e \leqslant 50$ and at different Hartmann numbers: $10 \leqslant R e \leqslant 50$ for $\mathrm{Ha}=0$; $15 \leqslant R e \leqslant 65$ for $H a=5.6,15 \leqslant R e \leqslant 85$ for $H a=8.4,15 \leqslant R e \leqslant 90$ for $H a=11.2$ and $15 \leqslant R e \leqslant 90$ for $H a=14$. The positions of the focus points $F_{C}$ are schematically shown in the sketches I and II. The flow structures relevant to the points $\mathrm{a}, \mathrm{b}$ and $\mathrm{c}$ are illustrated by the images shown in figure $6(a-c)$. Full dimensionless length of the cylinder is $L / d=4.46$.

of $S_{C}$ are displaced toward the cylinder in a narrow area of the $z$-coordinate, change. This is obviously linked with the reducing intensity of the head vortex for decreasing Reynolds number. The transition begins earlier if the value of the magnetic field is higher. In response to this the right-hand branches of the curves corresponding to moderate and high Reynolds numbers also approach the cylinder as the Hartmann number increases. This effect occurs, on the one hand, due to the suppression of the head vortex by the magnetic field which is perpendicular to its axis. On the other hand, it is due to an increasing effect of electromagnetic braking of the fluid above the cylinder for $N>1$, when the Ludford column begins to appear.

We identify three types of flow patterns in figure 7 according to the relative position of the singular points. The first type denoted by 'I' corresponds to the case of weak MHD interaction (i.e. $N \ll 1$ ) and is illustrated in figure 6(a). Here the head vortex with its axis perpendicular to the magnetic field is clearly seen. The focus point is situated far away from the surface of the cylinder. The second type of flow denoted by 'II' corresponds to moderate Reynolds numbers and interaction parameters $N>1$ at $H a=11.2$ and 14. In this case the head vortex decays and the intensity of the attached vortices behind the cylinder with the axes parallel to the magnetic field increases as can be seen in figure $6(b)$. The focus point is situated in the vicinity of the cylinder. The third type of flow denoted by 'III' corresponds to the highest possible Hartmann number $\mathrm{Ha}=14$ reached in our experiments and different Reynolds numbers obeying $N \gg 1$. In this case the head vortex is not developed, and the attached vortices 

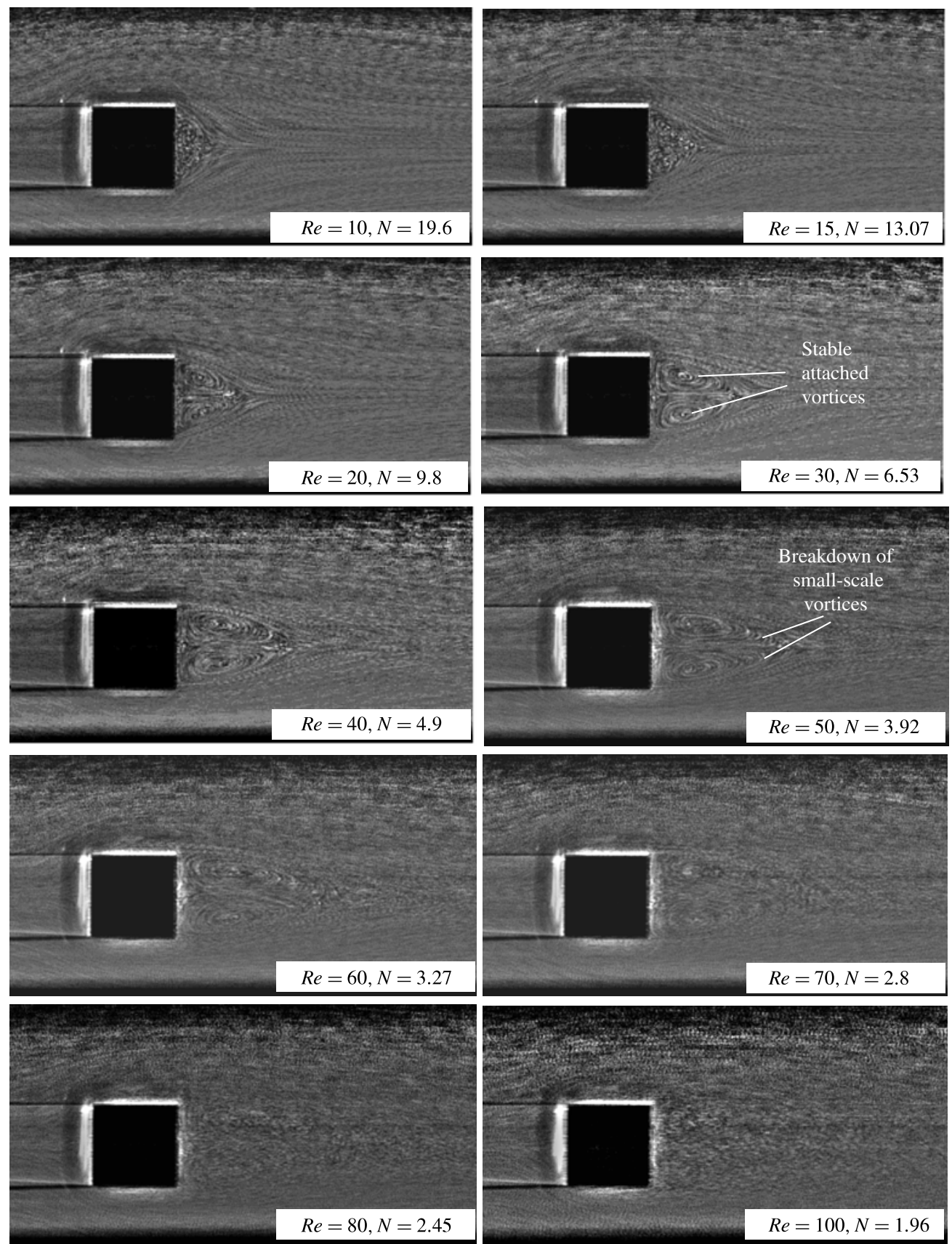

FIGURE 8. Visualization of the flow patterns past the cylinder in the horizontal plane $z=2.25$ for various Reynolds numbers and constant Hartmann number $H a=14$. The end of the cylinder was covered by black paint to enhance the clarity of the flow visualization.

parallel to the magnetic field exist in a narrow space behind the cylinder as shown in figure 6(c). Thus it can be seen that the described mechanism of transition from the flow configuration characterized by the head vortex (with axis oriented perpendicularly 
(a)

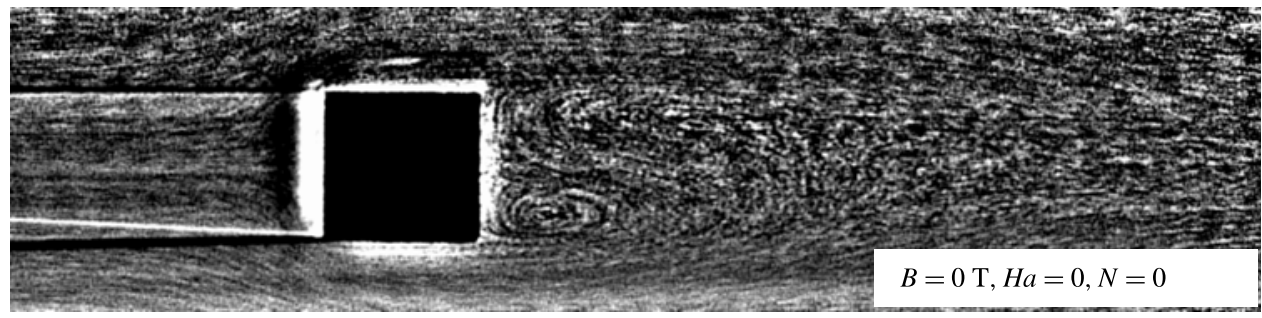

(b)

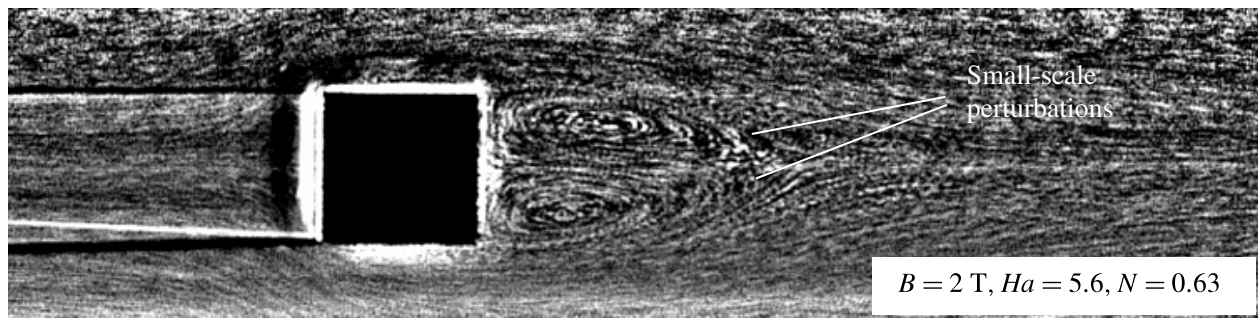

(c)

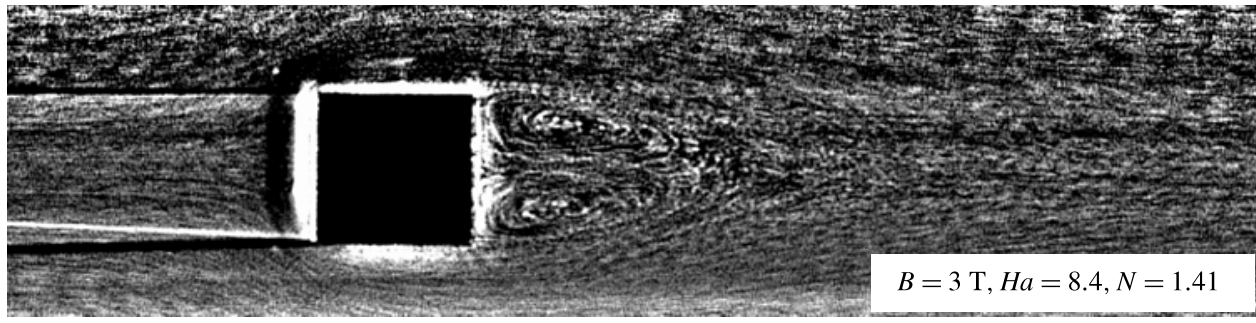

(d)

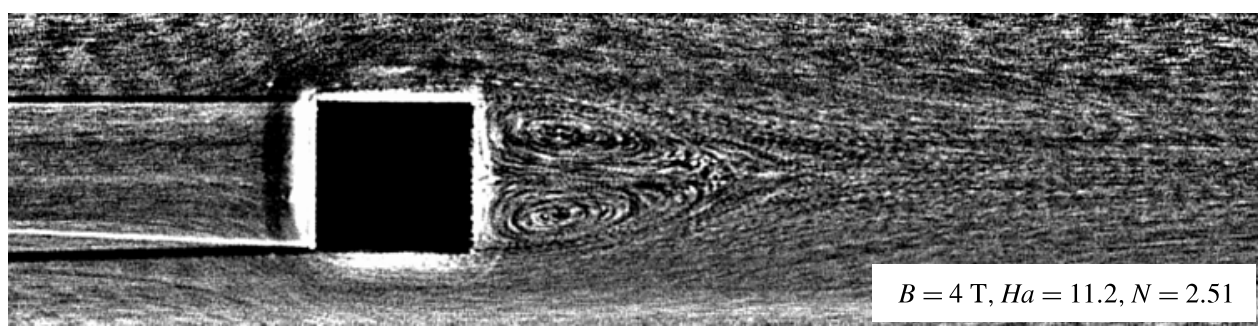

(e)

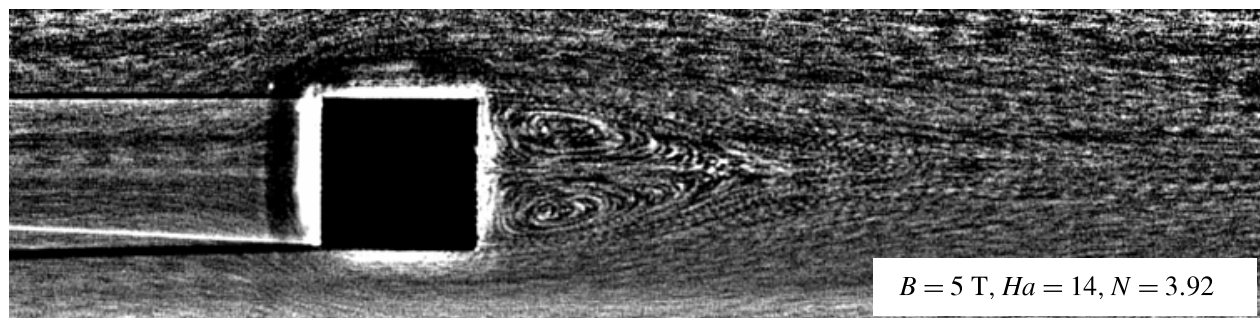

FIGURE 9. Visualization of the flow patterns past the cylinder in the horizontal plane $z=2.25$ for the Reynolds number $R e=50$ and various Hartmann numbers.

to the magnetic field) to the flow pattern presented by two attached vortices parallel to the field depends on both Reynolds $R e$ and Stuart $N$ numbers. 


\subsection{Flow structures for $10 \leqslant R e \leqslant 100$ in the plane perpendicular to the magnetic field}

In figure 8 we show selected flow patterns in the horizontal plane $z=2.25$ in the case of the highest possible value of the magnetic field equal to $5 \mathrm{~T}$ corresponding to $H a=14$. The Reynolds number is varied in the range $10 \leqslant R e \leqslant 100$. The images in figure 8 are obtained by integration of sequential PIV images over $5 \mathrm{~s}$ and represent the streamlines as time-averages of the fluid motion. At a small Reynolds number $R e=10$ (and $N=19.6$ ) a narrow stagnant zone is observed behind the cylinder. Upon increase of the Reynolds number the stagnant zone lengthens in the flow direction, and at $R e=15(N=13.07)$ the attached vortices are in prospect there. Upon a further increase of the Reynolds number we observe two distinct attached vortices as shown for $R e=20(N=9.8)$. The closed streamlines which correspond to the attached vortices are clearly seen. These structures are retained behind the cylinder in the Reynolds number range $20 \leqslant R e \leqslant 70(9.8 \leqslant N \leqslant 2.8)$. The domain of attached vortices continues to extend in the flow direction. The vortices are stable and symmetric up to the Reynolds number of $R e=60(N=3.27)$. The image for $R e=70(N=2.80)$ displays the presence of non-symmetrical vortices behind the cylinder which are prone to oscillations.

In our observations of this regime during the experiment we note that the vortices remain in the vicinity of the cylinder. It should be recognized that the shedding of attached vortices from a cylinder in ordinary hydrodynamics occurs at $R e=55$ (Zdravkovich 1997). The delay of the vortex shedding in our experiments is related to the Joule dissipation in the vortices caused by the angle between the direction of the magnetic field and the axes of vortices. If $R e \geqslant 80$, then the wake of the cylinder becomes perturbed and vortex shedding takes place.

We note that the development of the MHD instability takes place in two stages. Initially, in spite of the growth of Reynolds number, the attached vortices remain stable (for example for $R e<30$ in figure 8) and are stretched in the streamwise direction. Then the tail of the attached vortices begins to oscillate and to generate small-scale perturbations. They are visible in figure 8 at $R e=50$ and 60 . The dimensions of these perturbations are inversely proportional to the square root of the Hartmann number. This effect of two-stage onset of instability of the attached vortices is well identified in ordinary hydrodynamics (Zdravkovich 1997) and was already observed in the previous investigations with liquid metal (Kolesnikov \& Andreev 2000). Our present results confirm this peculiarity by optical visualization.

Figure 9 illustrates the stabilization of the attached vortices by the applied magnetic field. In this experimental run we keep the Reynolds number at the value $R e=50$ and change the magnitude of the magnetic field so that the Hartmann number is varied in a range $0 \leqslant H a \leqslant 14$. The exposure time used for these images is $2 \mathrm{~s}$, i.e. it is 2.5 times less than in the realizations shown in figure 8 . This exposure allows us to investigate unsteady behaviour. In the non-magnetic case $H a=0$ the attached vortices are separated from the cylinder as shown in figure $9(a)$. In particular, one can see that the image shows the instant when one of the vortices is inflated and is ready to separate from the cylinder whereas the second small vortex is developing. An application of the magnetic field acts on the flow in such a way that for magnetic fields between 2 and $3 \mathrm{~T}$, corresponding to $N=0.63$ and $N=1.41$ respectively, the vortex separation is terminated and the vortices become more stable as can be seen in figures $9(b)$ and $9(c)$.

However, the observations during the experiment also show that the vortices alternately fluctuate in the flow direction without a separation. This is clearly reflected in the figures. The stabilization under growing magnetic field is due to an 
intensification of Joule dissipation and increases even more due to the increasing Hartmann friction. In early experiments with cylinders in liquid metal flows (Andreev \& Kolesnikov 1997) the critical Reynolds number increased 70-fold in the presence of a magnetic field due to the Hartmann friction compared with the non-magnetic case. The pictures shown in figure 9 demonstrate a further enhancement of the stabilization effect as the magnetic field increases. For magnetic fields in the range between 4 and $5 \mathrm{~T}$ and corresponding to interaction parameters between $N=2.51$ and 3.92, the attached vortices are more stabilized and clearly pronounced in figures $9(d)$ and $9(e)$. Finally, it is interesting to mention that the von-Kármán-like vortex street observed at the onset of unsteadiness is very similar to that seen by Dousset \& Pothérat (2012) but that it seems much more stable at lower $H a$ than in the case with walls.

\section{Summary and conclusions}

In the present work we have performed a visualization of the flow around a truncated obstacle in a uniform axial magnetic field. The experiments were carried out by moving a partly submerged cylinder through a quiescent fluid. We used an aqueous solution of $\mathrm{H}_{2} \mathrm{SO}_{4}$ as the working fluid. Although the electrical conductivity of our working fluid was about $10^{4}$ times smaller than the conductivity of liquid metals, we were able to reach a sufficiently high electromagnetic interaction parameter $(N=39.2$ for $B=5 \mathrm{~T})$ so as to visualize the formation of the Ludford column. As commercially available PIV equipment is not designed to operate in the vicinity of a strong magnetic field, a low-cost self-made optical system had to be used to measure both the instantaneous and time-averaged flow fields. The ranges of the Reynolds number, the Hartmann number and the electromagnetic interaction parameters were $5 \leqslant R e \leqslant 100,0 \leqslant H a \leqslant 14$ and $0.31 \leqslant N \leqslant 39.2$. For these parameters we visualized the behaviour of the flow and the vortical structures past the cylinder, termed the "head vortex' generated by the flow above the cylinder and the attached vortices.

Our visualization showed that at $R e=5$ and $N=39.2$ a Ludford column clearly emerged from the cylinder and extended along the magnetic field direction.

In the non-magnetic case and for $R e=50$ we found that the head vortex and the attached vortices grow and are separated as known from ordinary hydrodynamics. The head vortex is perpendicular to the magnetic field and decays very quickly with increase of the field. More-stable attached vortices are observed for a small angle to the magnetic field and weakly interact with the magnetic field. It has been discovered that at $R e=50$ the attached vortices are stabilized at electromagnetic interaction parameters starting from $N=0.63(B=2 \mathrm{~T})$. For these parameters the separation of the vertical structure is stopped. Further growth of $N$ leads to a stabilization of attached vortices because of the stabilizing mechanism of Joule dissipation.

At fixed $H a=14$ and variable Reynolds number up to $R e=15$ a narrow stagnant area of fluid beyond the cylinder is observed. For $R e>15$ the stagnant area is elongated in the flow direction, and then at $R e=20$ one can see a well-defined system of two attached vortices the intensity of which grows with an increase the Reynolds number in the range between $R e=20$ and $R e=60$. Beginning at $R e=70$ the vortices undergo alternating oscillations along the flow, and at $R e>70$ they break down.

We conclude from our experiments that the combination of a high magnetic field of the order of $5 \mathrm{~T}$ with an electrolyte as a working fluid allows one to visualize MHD phenomena and to use optical flow measurement techniques for their quantitative characterization. We believe that future experiments in higher magnetic fields of the order of $10 \mathrm{~T}$ and the use of optical flow measurement techniques unaffected by 
high magnetic fields will offer the opportunity to study other MHD features such as Hartmann layers and Shercliff layers including their instabilities in much greater detail than with liquid metal experiments.

\section{Acknowledgements}

This work was supported by the Deutsche Forschungsgemeinschaft under grant number TH497/27-1. The authors are grateful to E. Roth for technical support and preparation of the experimental facility and to A. Pothérat and V. Dousset for illuminating discussions.

\section{REFERENCES}

Andreev, O. \& KolesniKov, Yu 1997 MHD instabilities at transverse flow around a circular cylinder in an axial magnetic field. In Third International Conference on Transfer Phenomena in Magnetohydrodynamic and Electroconducting Flows, pp. 205-210. Aussois.

Branover, G. G., Gel'Fgat, Yu. M., Turuntaev, S. V. \& Tsinober, A. B. 1969 Effect of a transverse magnetic field on velocity perturbations behind a circular cylinder swept by an electrolyte. Magnetohydrodynamics 5 (3), 41-46.

DAVIDSON, P. A 1997 The role of angular momentum in the magnetic damping of turbulence. J. Fluid Mech. 336, 123-150.

Davidson, P. 1999 Magnetohydrodynamics in materials processing. Annu. Rev. Fluid Mech. 31, 273-300.

Dousset, V. \& Pothérat, A. 2008 Numerical simulations of a cylinder wake under a strong axial magnetic field. Phys. Fluids 20, 017104.

Dousset, V. \& Pothérat, A. 2010 Formation mechanism of hairpin vortices in the wake of truncated square cylinder in a duct. J. Fluid Mech. 653, 519-536.

Dousset, V. \& PothÉrAT, A. 2012 Characterisation of the flow around a truncated cylinder in a duct in a spanwise magnetic field. J. Fluid Mech. 691, 341-367.

Frank, M., BARLeON, L. \& MÜLleR, U. 2001 Visual analysis of two-dimensional magnetohydrodynamics. Phys. Fluids 13 (8), 2287-2295.

Greenspan, H. P. 1969 The Theory of Rotating Fluids. Cambridge University Press.

Hunt, J. C. R., Abell, C. J., Peterka, J. A. \& Woo, H. 1978 Kinematical studies of the flows around free or surface-mounted mounted obstacles; applying topology to flow visualization. J. Fluid Mech. 86, 179-200.

Hunt, J. C. R. \& Leibovich, S. 1967 Magnetohydrodynamic flow in channels of variable cross-section with strong transverse magnetic fields. J. Fluid Mech. 28, 241-260.

Hunt, J. C. R. \& Ludford, G. S. S. 1968 Three-dimensional MHD duct flows with strong transverse magnetic fields. Part 1. Obstacles in a constant area channel. J. Fluid Mech. 33, 693-714.

Josserand, J., Marty, P. \& Alémany, A. 1993 Pressure and drag measurements on a cylinder in a liquid metal flow with an aligned magnetic field. Fluid Dyn. Res. 11, 107-117.

Kolesnikov, Yu. \& ANDREEV, O. 1997 Heat transfer intensification promoted by vortical structures in a closed channel under magnetic field. J. Expl Therm. Fluid Sci. 15 (2), 82-90.

Kolesnikov, YU. \& AndREeV, O. 2000 Two scaling flow around a cylinder in axial magnetic field. In The 4th International Conference: MHD at Dawn of 3rd Millennium, pp. 33-38. Giens.

Kolesnikov, Yu. B. \& Tsinober, A. B. 1972 Two-dimensional turbulent flow behind a circular cylinder (experiment). Magnetohydrodynamics 8 (3), 300-307.

LAhjomri, J., CAPÉRAn, P. \& AlÉmany, A. 1993 The cylinder wake in a magnetic field aligned with the velocity. J. Fluid Mech. 253, 421-448.

LUdFORD, G. S. S. 1961 The effect of a very strong magnetic cross-field on steady motion through a slightly conducting fluid. J. Fluid Mech. 10, 141-155.

Moreau, R. 1990 Magnetohydrodynamics. Kluwer. 
MÜlleR, U. \& BÜHLER, L. 2010 Magnetofluiddynamics in Channels and Containers. Springer.

Mutschke, G., Gerbeth, G., Shatrov, V. \& Tomboulides, A. 1997 Two- and three dimensional instabilities of the cylinder wake in an aligned magnetic field. Phys. Fluids 9 (11), 3114-3116.

Pothérat, A., Sommeria, J. \& Moreau, R. 2000 An effective two-dimensional model for MHD flows with transverse magnetic field. J. Fluid Mech. 424, 75-100.

Raffel, M., Willert, C. E. \& Kompenhans, J. 1998 Particle Image Velocimetry. Springer.

SHERCLIFF, J. A. 1953 Steady motion of conducting fluids in pipes under transverse magnetic fields. Proc. Camb. Phil. Soc. 49, 136-144.

ShERCLIFF, J. A. 1962 The Theory of Electromagnetic Flow-measurement. Cambridge University Press.

Shercliff, J. A. 1975 Some duct flow problems at high Hartmann number. Z. Angew. Math. Phys. 26, 537-548.

Sommeria, J. \& Moreau, R. 1982 Why, how, and when, MHD turbulence becomes two-dimensional. J. Fluid Mech. 118, 507-518.

Takizawa, Y, Matsuda, A., Sato, S., Abe, T. \& Konigorski, D. 2006 Experimental investigation of the electromagnetic effect on a shock layer around a blunt body in a weakly ionized flow. Phys. Fluids 18, 117105.

Zaytsev, I. D. \& Aseyev, G. G. 1992 Properties of Aqueous Solutions of Electrolytes. CRC.

Zdravkovich, M. M. 1997 Flow Around Circular Cylinders. Fundamentals, vol. 1, Oxford University Press. 